\title{
High-latitudes magnetic substorms under different types of the solar wind large-scale structure
}

\author{
Irina Despirak ${ }^{1}$, Andris Lubchich ${ }^{1}$, Natalia Kleimenova ${ }^{2}$ \\ ${ }^{1}$ Polar Geophysical Institute, Apatity, Murmansk region, Russia, \\ ${ }^{2}$ Schmidt Institute of the Earth Physics, Moscow, Russia
}

e-mail: (despirak@gmail.com)

Accepted date: January 26, 2018

\begin{abstract}
Based on the IMAGE magnetometer network data, OMNI database of the solar wind and Interplanetary Magnetic Field (IMF) parameters, and the catalog of the large-scale solar wind types (ftp://ftp.iki.rssi.ru/omni/), the comparative analysis of the interplanetary conditions of the high-latitude substorm appearance has been carried out. We analyzed substorms observed at the meridional chain (TAR-NAL) of IMAGE magnetometer stations in 1995-1996, and 1999-2000. According to our previous study (Despirak et al., 2014; 2016; 2017), we divided the considered substorms into two types. First type - the substorms which propagate from the auroral latitudes $\left(<70^{\circ} \mathrm{CGC}\right)$ to polar geomagnetic ones $\left(>70^{\circ} \mathrm{CGC}\right)$ (so called "expanded" substorms); the second type - the substorms which are observed only at the latitudes higher $\sim 70^{\circ}$ CGC under the absence of simultaneous geomagnetic disturbances at the latitudes below $70^{\circ}$ (so called "polar" substorms). The 202 "expanded" and 186 "polar" substorms have been selected during the four considered years. It is shown that the "expanded" substorms are mainly (in 74.7\% of the events) observed while the high-speed recurrent streams (FAST) and the regions of the plasma compression before these streams (CIR) occur. For $18.3 \%$ of the events, such substorms are observed during the interplanetary displays of coronal mass ejections (SHEATH and EJECTA). Contrary to that, the "polar" substorms occur mainly (in 67.2\% of the events) during the slow flows and heliospheric current sheet (SLOW and HCS); and for $18.8 \%$ of the events, they occur during the SHEATH, EJECTA and MCs, which were observed in the background of the slow solar wind. Thus, the space weather conditions control the type of the developed highlatitude magnetic substorms in the Earth's magnetosphere.
\end{abstract}

(c) 2018 BBSCS RN SWS. All rights reserved

Key words: magnetic clouds, high speed streams, CIR- and Sheath regions, substorms

\section{Introduction}

Many researchers considering different disturbances in the Earth's magnetosphere compared these with specific types of the solar wind (e.g. Huttunen, and Koskinen, 2004; Pulkkinen et al., 2007; Turner et al., 2009; Guo et al., 2011; Yermolaev et al., 2014). It is common knowledge that there are different large-scale structures and flows inside the solar wind (Krieger et al., 1973; Burlaga et al., 1982; Pudovkin, 1996) as the reflection of the large-scale structure of the solar corona. On the other hand, in order to describe the complex dynamics of the solar wind processes, simplified approaches are needed that consider a set of "typical phenomena". Indeed, the solar wind contains the propagation in the interplanetary space different regions or stream types. Each "type" characterized by certain values of the solar wind parameters, and these values can vary slightly within the "types". It is important to apply a definite classification of the large-scale flows and structures in the solar wind for studying the processes in the system "Sun-solar wind-magnetosphere".

Usually, these flows are considered as quasistationary or sporadic structures (Pudovkin, 1996). There are three quasi-stationary solar wind types - fast and slow solar wind, generated in the coronal holes and coronal streamer belts, and the heliospheric current sheet (HCS). The phenomena, associated with the interplanetary manifestations of coronal mass ejections (CME), which include the magnetic clouds (MCs) and EJECTA are assigned to sporadic flows. The term "sporadic" also covers the structures generating in the interplanetary medium in the spread of the solar wind. There are regions of the plasma compression in fronts of the fast recurrent flows (CIR - Corotating Interaction Regions), as well as in front of the magnetic clouds and EJECTA (SHEATH regions) and also the direct and reverse shock waves (IS and ISa) (e.g. Klein and Burlaga, 1982; Schwenn, 2006; Sheeley and Harvey, 1981; Cane and Richardson, 2003; Gopalswamy, 2006). In the present paper, we used the classification of the solar wind types, described by Yermolaev et al., (2009) which is based on the OMNI data and the catalog of the large-scale solar wind phenomena (ftp://ftp.iki.rssi.ru/omni/)/. Some basic characteristics of each type of solar wind are presented in the section "Data".

It should be noted that there are several other catalogs of the solar wind types, e.g. ICME catalog (Richardson and Cane, 2010), MC catalog (https://wind.nasa.gov/mfi/mag_cloud_publ.html),

ISTP Solar Wind Catalog (http://WWWspof.gsfc.nasa.gov/scripts/swcat/

Catalog_events.html). However, in our opinion, the types of the solar wind are presented the most widely and reasonable in the catalog (Yermolaev et al., 2009), moreover, the time range of the dates of this catalog is the largest one - from 2000 to 2016.

Different types of the solar wind are characterized by the different degrees of the effect in the Earth's magnetosphere, or by the different geoeffectiveness (e.g. Denton et al., 2006; Huttunen et al., 2006; Pulkkinen et al., 2007; Turner et al., 2009; Longden et al, 2008; Despirak et al., 2009; McPherron et al., 2009). It is generally accepted, that the sources of geomagnetic storms are certain flows in the solar wind - the high speed streams from the coronal magnetic holes and the sporadic flows, related to the coronal mass ejections (CMEs), as well as the regions of the 
compressed plasma in the front of these flows, the CIR and Sheath-regions. The geomagnetic storms caused by the different types of the solar wind drivers differ by their characteristics: the intensity, duration of the recovery phase etc. (e.g. Borovsky and Denton, 2006; Yermolaev et al., 2010). The substorms, observed under different interplanetary conditions, e. g., during storms, caused by the different solar wind types, and during the quiet conditions, also differ by their characteristics (the intensity, formation and development of the auroral bulge, sizes in geomagnetic latitude and longitude etc.) (Tsurutani et al., 2004, 2006; Clausen et al., 2013, Wang et al., 2005; Tanskannen et al., 2011). However, despite a large number of papers devoted to the study of substorms under different interplanetary conditions, they do not give a complete picture, because more of them presented or only an analysis of one individual selected event, or did not involve the long continuous series of the solar wind variations.

In this paper, we consider an effect of a large-scale solar wind structure on the appearance of the substorms at the high geomagnetic latitudes. We combine the data of the ground-based IMAGE magnetometers, OMNI data base for the interplanetary medium parameters and the catalog of the large-scale solar wind phenomena (ftp://ftp.iki.rssi.ru/omni/). In our study, we consider only substorms, observed at the geomagnetic latitudes higher $\sim 70^{\circ}$ CGC (i.e. above BJN IMAGE station location). As in previous studies (Despirak et al., 2014; 2016; 2017), we divided the high-latitude substorms into 2 types. The first type includes the substorms which propagate from auroral latitudes $\left(<70^{\circ}\right)$ to polar geomagnetic latitudes $\left(>70^{\circ}\right)$ (called "expanded" substorms, according to an expanded auroral oval location); the second type includes the substorms which are observed only at latitudes higher $\sim 70^{\circ}$ in the absence of simultaneous geomagnetic disturbances below $70^{\circ}$ (termed here as "polar" substorms, according to a contracted oval).

In our previous works we considered dependences of these two types of high-latitude substorms on the solar wind parameters, geomagnetic conditions (Despirak et al., 2014), seasons and on the solar cycle activity (Despirak et al., 2016, 2017). It was shown that, independently of the solar cycle, the "polar" substorms show behavior opposite to the "expanded" ones (e.g. seasonal variations, dependence on PC-index etc.). It was found that there were the certain space weather conditions leading to the occurrence of the "polar" substorms or the "expanded" substorm. It was shown that the most important parameters controlling the type of the substorms are the solar wind velocity, electric field, and, probably, the IMF By sign. The aim of this paper is to study the relationship between a certain type of the solar wind and the appearance of these two types of high-latitude substorms.

\section{Data}

We apply the solar wind type classification according to the OMNI data base and the catalog of large-scale solar wind phenomena (ftp://ftp.iki.rssi.ru/omni/). In this catalog, there are distinguished three quasi-stationary types of the solar wind, five perturbed types, and shock waves. Several general characteristics of each type are presented below.

The Heliospheric Current Sheet (HCS) is associated with the sector structure of the solar wind, separating regions of the interplanetary magnetic field directed to the Sun and from the Sun. This area is characterized by the simultaneous change of the signs of the magnetic field components $\mathrm{Bx}_{\mathrm{x}} \mathrm{B}_{\mathrm{y}}$ and consists of cold plasma with low speed and high density, as well as a decreased magnetic field.

The Slow stream (SLOW), observed over the equatorial streamer belt of the Sun, is the denser and colder plasma stream with lowered values of the velocity $(V<450 \mathrm{~km} / \mathrm{s})$. The Fast stream (FAST), occurs above the polar coronal holes with open magnetic field lines, is the hot and rarefied plasma stream with enhanced values of the velocity $(V>450 \mathrm{~km} / \mathrm{s})$. It is mentioned, that there is no sharp boundary between the SLOW and the FAST plasma. Therefore, the boundary between the SLOW and the FAST streams can be made conditionally. Thus, in the catalog the plasma streams with speed $V<450 \mathrm{~km} / \mathrm{s}$ are considered as SLOW streams, and plasma streams with speed $V>450 \mathrm{~km} / \mathrm{s}$ are considered as the FAST streams.

The Interplanetary coronal mass ejections CME (EJECTA) are large-scale heliospheric transients (Kilpua et al., 2017) that contain the magnetic field in the form of a flux rope structure where the magnetic pressure is higher than the thermal pressure $(\beta \ll 1)$. The Magnetic cloud (MC) is a subclass of EJECTA which differs by a higher and more regular magnetic field. When an CME is sufficiently faster than the preceding solar wind, a shock wave develops ahead of the CME. The turbulent compressed plasma region located between the shock and the CME body (the MC/EJECTA) is called the SHEATH.

The corotating interaction region (CIR) where a fast stream from a coronal hole overtakes a leading slow stream are regions, where the plasma is characterized by enhanced values of the density, temperature and magnetic field magnitude, and $\beta>1$. The rarefied plasma region (RARE) is the region after a fast plasma steams with small values of density $N<1 \mathrm{CM}^{-3}$ (after FAST or EJECTA/MC).

It should be noted that there are a number of papers describing the average values of the parameters in these types of solar wind, which confirms the accepted classification with small variations in the methods of identification and differences in the selected interval (e.g. Thatcher and Muller, 2011; Mitsakou and Moussas, 2014).

In this study, we used the 10-s sampled IMAGE magnetometer data. The high-latitude substorms were manually identified at the magnetograms from the TAR-NAL meridional chain (Tartu (TAR), CGC lat. = $54.47^{\circ}$ - Ny Ålesund (NAL), CGC lat. $=75.25^{\circ}$ ). Note, that many clear examples of the "polar" and "expanded" substorms observed at the IMAGE meridional chain were considered in the recent papers by Despirak et al. $(2014,2017)$. In the presented paper, we analyzed substorms at the high latitudes in 1995, 1996, 1999 and 2000. Total 202 "expanded" and 186 "polar" substorms were selected during 4 years. 


\section{Results}

We compared the appearance of two types of high-latitude substorms - "polar" and "expanded" with the simultaneous records of the large-scale structure of the solar wind. The obtained results are presented in Tables 1 and 2. The number of "expanded" substorms, observed under different types of the solar wind, are shown in Table 1, where the number of the substorms observed during the different solar wind types on the background streams is shown in the left columns, the total percent of the substorms number during certain types of the solar wind is given in the right columns. It is seen that the "expanded" substorms are observed mainly during the high-speed recurrent streams (FAST) (60.9\% of events). It should be mentioned that high speed streams are usually denoted HSS, but here we used the terminology of the solar wind types according to Yermolaev et al. (2009).

Table 1 demonstrates that the "expanded" substorms were observed during two regions of the plasma compression before fast streams: before the recurrent high speed streams (CIR) and before magnetic clouds (MC) or Ejecta (SHEATH) $119.2 \%$ of events). It is interesting that the "expanded" substorms were observed not only during the CIRs and SHEATHS regions on the background of a fast stream (CIR, FAST; SHEATH, FAST), but also during these regions on the background of a slow stream $(<450 \mathrm{~km} / \mathrm{s})$ (CIR, SLOW; Sheath, SLOW). This fact demonstrates that other common parameters, characterizing the CIR and Sheath regions, are also favorable parameters for the appearance of the "expanded" substorms, namely, high values of the density, pressure and temperature of the solar wind. Table 1 shows as well that the "expanded" substorms were observed during the interplanetary displays of coronal mass ejections, namely, during the EJECTA (12.9\%). Moreover, all EJECTA events were observed against the background of the high-speed flows (Ejecta, FAST). We noted that some events of the "expanded" substorms (only 3.5\% of cases) were recorded during the slow flows (SLOW) and heliospheric current sheet (SLOW, HCS), and some events ( $3.5 \%$ of cases) - without a certain flow.

The distributions of the "polar" substorms, observed under different types of the solar wind, are given in Table 2. The format of Table 2 is the same as Table 1 . It is seen that the "polar" substorms occur mainly during the solar wind slow flows (SLOW) and heliospheric current sheet (SLOW, HCS) (67.2\% of events). The main feature of these solar wind types is the low values of the solar wind velocity, and additionally to the simultaneous changes of the $B_{x}$ and $B_{Y}$ IMF signs, the high density of the solar wind. Some of the "polar" substorms ( 12.9\% of cases) were observed at the end of the high-speed recurrent stream (FAST) when the solar wind velocity decreased (from high to low values). It should be pointed out that at the beginning of the high speed streams, the "polar" substorms occurred rarer than at the end of the streams. We found only one event which was recorded at the beginning of the high speed stream (FAST, start of stream). Unexpectedly, it was found that the "polar" substorms were recorded also during the interplanetary displays of the coronal mass ejections, namely, during EJECTA ( $16.7 \%$ of events). However, as a rule, all
Table 1. The number of "expanded" substorms, recorded under different types of the solar wind. The left column lists certain solar wind types and the background stream and the number of the correspondent substorms observed under given condition, the right column shows the total percent of substorms number during certain types of the solar wind.

\begin{tabular}{|c|c|c|c|}
\hline $\begin{array}{l}\text { Type of solar } \\
\text { wind and the } \\
\text { background } \\
\text { stream }\end{array}$ & $\begin{array}{l}\text { Number of } \\
\text { "expanded" } \\
\text { substorms }\end{array}$ & $\begin{array}{l}\text { Type of } \\
\text { solar wind }\end{array}$ & $\begin{array}{c}\text { Total, } \\
\%\end{array}$ \\
\hline CIR, SLOW & 5 & \multirow{2}{*}{ CIR } & \multirow{2}{*}{13.8} \\
\hline CIR, FAST & 23 & & \\
\hline FAST & 121 & \multirow{2}{*}{ FAST } & \multirow{2}{*}{60.9} \\
\hline FAST, HCS & 2 & & \\
\hline EJECTA, FAST & 25 & \multirow{2}{*}{ EJECTA } & \multirow{2}{*}{12.9} \\
\hline EJECTA, SLOW & 1 & & \\
\hline SHEATH, SLOW & 5 & \multirow{2}{*}{ SHEATH } & \multirow{2}{*}{5.4} \\
\hline SHEATH, FAST & 6 & & \\
\hline MC & $\mathbf{0}$ & MC & $\mathbf{0}$ \\
\hline SLOW & 3 & \multirow{2}{*}{ SLOW } & \multirow{2}{*}{3.5} \\
\hline SLOW, HCS & 4 & & \\
\hline $\begin{array}{l}\text { without certain } \\
\text { SW types }\end{array}$ & 7 & $\begin{array}{c}\text { without } \\
\text { certain SW } \\
\text { types }\end{array}$ & 3.5 \\
\hline
\end{tabular}

Table 2. The same as in Table 1 for the "polar" substorms.

\begin{tabular}{|c|c|c|c|}
\hline $\begin{array}{l}\text { Type of solar } \\
\text { wind }\end{array}$ & $\begin{array}{l}\text { Number of } \\
\text { "polar" } \\
\text { substorms }\end{array}$ & $\begin{array}{l}\text { Type of solar } \\
\text { wind }\end{array}$ & $\begin{array}{c}\text { Total, } \\
\%\end{array}$ \\
\hline CIR, SLOW & 2 & \multirow{2}{*}{ CIR } & \multirow{2}{*}{1.07} \\
\hline CIR, FAST & $\mathbf{0}$ & & \\
\hline $\begin{array}{c}\text { FAST, end of } \\
\text { stream }\end{array}$ & 23 & \multirow{2}{*}{$\begin{array}{l}\text { End and start } \\
\text { of the FAST }\end{array}$} & \multirow{2}{*}{12.9} \\
\hline $\begin{array}{c}\text { FAST, start of } \\
\text { stream }\end{array}$ & 1 & & \\
\hline FAST, HCS & $\mathbf{0}$ & FAST & $\mathbf{0}$ \\
\hline EJECTA, FAST & $\mathbf{0}$ & \multirow{2}{*}{ EJECTA } & \multirow{2}{*}{16.7} \\
\hline EJECTA, SLOW & 31 & & \\
\hline SHEATH & $\mathbf{0}$ & \multirow{2}{*}{ SHEATH } & \multirow{2}{*}{1.6} \\
\hline SHEATH, FAST & 3 & & \\
\hline SHEATH, MC & $\mathbf{0}$ & \multirow{2}{*}{ MC } & \multirow{2}{*}{0.53} \\
\hline MC,SLOW & 1 & & \\
\hline SLOW & 92 & \multirow{2}{*}{ SLOW } & \multirow{2}{*}{67.2} \\
\hline SLOW, HCS & 33 & & \\
\hline $\begin{array}{l}\text { without certain SW } \\
\text { types }\end{array}$ & $\mathbf{0}$ & $\begin{array}{c}\text { without } \\
\text { certain } S W \\
\text { types }\end{array}$ & $\mathbf{0}$ \\
\hline
\end{tabular}

EJECTA events were developed against the background of the low-speed streams (EJECTA, SLOW). We found only one event of "polar" substorm, which occurred during magnetic cloud (MC) $10.53 \%$ of events). But in that case, it was the magnetic cloud under the low solar wind velocity, and the considered substorm has been registered under positive values of the BZ component of the IMF. 


\section{Discussion}

We found that different types of the high-latitude magnetic substorms are observed under different conditions of the space weather, determined by the large-scale structure of the solar wind.

It is well-known that different types of the solar wind are characterized by the different combinations of the geoeffective parameters (e.g., Burlaga et al, 1982; Krieger et al., 1973; Klein and Burlaga, 1982; Tsurutani et al, 2006; Mitsakou and Moussas, 2014) As an example, the high speed streams from coronal holes (FAST) demonstrate the high values of the solar wind velocity ( $v>450 \mathrm{~km} / \mathrm{s}$ ) and temperature but low density. The slow flows (SLOW) show low values of the solar wind velocity ( $v<450 \mathrm{~km} \mathrm{/} \mathrm{s)} \mathrm{and} \mathrm{the} \mathrm{simultaneously}$ enhanced density under the low temperature (Yermolaev et al, 2009). Therefore, regarding an influence of the different solar wind types, we assume effects of the different combinations of the geoeffective parameters, typical for a particularly given type of the solar wind structure.

Previously, Despirak et al., (2014; 2016; 2017) reported that the high-latitude "expanded" substorms are observed under high values of the solar wind speed, and the"polar" substorms - under the low ones.

It should be noted that many studies provided details about the behavior of the westward electrojet and auroras at extremely high latitudes (Loomer and Gupta 1980; Gussenhofen 1982; Nielsen et al. 1988; Weatherwax et al. 1997; Kuznetsov et al. 2001; Yahnin et al. 2004). The substorms, which propagate to such high latitudes, were often considered as a separate type of substorms and were called "high-latitude" substorms (Sergeev et al. 1979; Dmitrieva and Sergeev 1984; Despirak et al. 2008) or the "substorms with large poleward expansion" (Kuznetsov et al., 2001). In the early works, a rapid poleward substorm expansion was considered as a different substorm phenomenon - a "poleward leap" (Hones et al. 1985; Pytte et al. 1978; Rostoker 1986). A number of authors showed that the "high-latitude" substorms occur more frequently under high solar wind velocity and during the solar cycle minimum when high speed recurrent streams from the coronal magnetic holes prevail (Sergeev et al. 1979; Dmitrieva and Sergeev 1984, Despirak et al. 2011, 2014). During solar cycle maximum, when magnetic clouds become the most typical solar wind disturbance, the substorms do not reach the polar latitudes, and so-called the "high-latitude" substorms were rarely observed (Despirak et al. 2009).

In our work we have confirmed that, the "expanded" substorms (which in early works were called "high-latitude" substorms), occur during the high values of the solar wind speed. We underline that these high values were associated mostly with FAST streams type, rather than with MC or EJECTA. Moreover, we have also found some additional geoeffective factors, e.g. the solar wind density, temperature, magnitude, regularity of the magnetic field, and the value of the parameter $\beta$ (the relationship between thermal and magnetic pressure) which characterize each given type of the solar wind structure. Sometimes, the influence of these additional parameters of the solar wind could lead to the appearance of the "expanded" substorms even under the relatively low values of the solar wind velocity, e.g. during the Sheath and CIR regions with a high solar wind density, pressure, and magnetic field magnitude and variability.

We also confirmed the result, obtained earlier, that the "polar" substorms occur under low values of the solar wind speed. New findings showed that these substorms are observed not only during the SLOW streams, but also during the ELECTA/MC and at the end or at the beginning of the FAST streams. However, it is mentioned that the ELECTA/MC events, as a rule, were developed against the background of the lowspeed streams SLOW.

\section{Summary}

The comparison of the high-latitude magnetic substorms appearance with the solar wind large-scale structure demonstrated:

1. The "expanded" substorms, i.e. substorms propagated from the auroral to polar latitudes, are observed during:

$>$ the high speed streams from coronal magnetic holes (FAST);

$>$ the plasma compressed regions (CIR, SHEATH) before high speed stream (FAST) or before MC/EJECTA, respectively;

$>$ interplanetary coronal mass ejection (EJECTA), which were observed on background of fast stream of the solar wind (FAST)

2. The "polar" substorms, i.e. substorms which are developed only at latitudes near the polar cap boundary, are observed during:

$>$ the slow streams (SLOW) and the slow streams which occur on the background of a helioshperic current sheet (SLOW, HCS);

$>$ at the end or at the beginning of the high speed stream (FAST), when the solar wind velocity already not reaches high values;

> the interplanetary coronal mass ejections, which occur on the background of the slow speed stream of the solar wind (EJECTA, SLOW).

\section{Acknowledgements.}

The WIND data used in this study were taken from OMNI web site

http://cdaweb.gsfc.nasa.gov/cdaweb/istp_public/. We are grateful to J. N. King and N. Papatashvili, the heads of the experiments conducted with these instruments.

This study was partly supported by Program No 28 of the Presidium of the RAS. 


\section{References:}

Borovsky, J.E. and Denton, M.H.: 2006, J. Geophys. Res., 28, 121. Burlaga, L.F., Klein, L., Sheeley, N.R. Jr., Michels, D.J., Howard, R.A., Koomen, M.J., Schwenn, R., Rosenbauer, H.:1982, Geophys. Res. Lett. 9, 1317.

Cane, H.V. and Richardson, I.G.:2003, J. Geophys. Res., 108. doi: 10.1029/2002JA009817.

Clausen, L. B. N., Milan, S.E., Baker, J. B. H., Ruohoniemi, J. M. Glassmeier, K.-H., Coxon, J. C., Anderson, B. J.: 2013, J. Geophys. Res., 118, 2958- 2969, doi:10.1002/jgra.50308.

Denton, M.H., Borovsky, J. E., Skoug, R.M., Thomsen, M.F., Lavraud, B., Henderson, M.G., McPherron, R.L., Zhang, J.C., Liemohnet, M.W.: 2006, J. Geophys. Res., 111. A07S07. doi:10.1029/2005JA011436.

Despirak, I.V., Lyubchich, A.A., Biernat, H.K., Yakhnin, A.G.: 2008, Geomag. Aeron 48, 284-292.

Despirak, I.V., Lubchich, A.A., Yahnin, A.G., Kozelov, B.V., Biernat, H.K.: 2009, Ann. Geophys. 27 (5), 1951.

Despirak, I.V., Lyubchich, A.A., Kleimenova, N.G.: 2014, Geomag. Aeron. 54(5), 575

Despirak, I.V., Lubchich, A.A., Kleimenova, N.G.: 2016, Sun and Geosphere, 11(2), 101

Despirak, I.V., Lubchich, A.A., Kleimenova, N.G.: 2017, J. Atmos. Sol. Terr. Phys., doi: 10.1016/j.jastp.2017.09.011.

Dmitrieva, N.P., Sergeev, V.A.: 1984, Magnitos. Issled. (in Russian) 3, 58-66.

Gopalswamy, N.: 2006, Space Sci. Rev., 124, 145-168. doi: 10.1007/s11214-006-9102-1. 2006.

Gussenhoven, M.S.: 1982, J. Geophys. Res., 87, 2401-2412.

Guo, J., Feng, X., Emery, B.A., Zhang, J., Xiang, C., Shen, F., Song, W.: 2011, J. Geophys. Res., 116. P. A05106. doi:10.1029/2011JA016490.

Hones, E.W. Jr.:1985, J. Geophys. Res., 90, 5333-5337.

Huttunen. K.E.J. and Koskinen, H.E.J.: 2004, Ann. Geophys., 22, 1729.

Huttunen, K. E. J., Koskinen, H. E. J., Karinen, A., Mursula, K.: 2006, Geophys. Res. Lett., 33, L06107. doi:10.1029/2005GL024894.

Kilpua, E., Koskinen, H. E. J., Pulkkinen, T. I.: 2017, Living Rev. Sol. Phys., 14(5), doi.org/10.1007/s41116-017-0009-6.

Krieger, A.S., Timothy, A.F., Roelof, E.C.:1973, Sol. Phys., 29 (2), 505.

Klein, L.W. and Burlaga, L.F.: 1982, J. Geophys. Res., 87 (A2), 613.

Kuznetsov, S., Lazutin, L.L., Rosenberg. T., Borovkov, L., Gotselyuk, Yu., Weatherwax, A.: 2000, In: Wilson A (ed) Proceedings of the 5th International Conference on Substorms, Conference on Substorms, St. Petersburg, Russia, 16-20 May 2000 (ESA SP-443, July 2000), 511-514.

Loomer, E.I., Gupta, J.C.: 1980, J. Atmos. Sol.-Terr. Phys. 42, 645-652.

Longden, N., Denton, M.H., Honary, F.: 2008, J. Geophys. Res., 113. A06205. doi:10.1029/2007JA012752.

Mitsakou, E., Moussas, X.: 2014, Solar Phys., 289, 3137-3157. doi:10.1007/s11207-014-0505-y.

McPherron, R.L., Kepko, L., Pulkkinen, T.I., Hsu, T.S.,Weygand, J.W., Bargatze, L.F. : 2009, Ann. Geophys., 27., 3165.

Nielsen, E., Bamber. J., Chen. Z.-S., Brekke, A., Egeland, A., Murphree, J.S., Venkatesan, D., Axford, W.I.: 1988, Ann. Geophys., 6, 559-572.

Pytte, T., McPherron, R.L., Kivelson. M.S., West, H.I. Jr, Hones, E.W. Jr.: 1978, J. Geophys. Res. 83, 5256-5268.

Pudovkin, M.I.: 1996, Soros Educational Journal, 12, 87.

Pulkkinen, T.I., Partamies, N., Huttunen, K.E.J., Reeves, G.D., Koskinen, H.E.J.: 2007, Geophys. Res. Lett., 34, L02105. doi:10.1029/2006GL027775.

Richardson, I.G., Cane, H.V.: 2010, Solar Phys., 264,189-237, doi: 10.1007/s11207-010-9568-6.

Rostoker, G.: 1986, J. Geophys. Res. 91, 5879-5880.

Schwenn, R.: 2006, Space Sci. Rev., 124, 51-76. doi: 10.1007/s11241-006-9099-5.

Sheeley, N.R. Jr. and Harvey, J.W.: 1981. Solar Phys., 70 (2), 237.

Sergeev, V.A., Yakhnin, A.G., Dmitrieva, N.P.: 1979, Geomag Aeron (in Russian) 19, 1121-1122.
Tanskanen, E. I., Pulkkinen, T. I., Viljanen, A., Mursula, K., Partamies, N., Slavin, J. A.: 2011, J. Geophys. Res., 116, A00134, doi:10.1029/2010JA015788.

Thatcher, L.J., Muller, H.-R.: 2011, J. Geophys. Res., 116, A12107. doi:10.1029/2011JA017027.

Tsurutani, B.T., Gonzales, W.D., Guarnieri, F., Kamide, Y., Zhou, X., Arballo, J.K.: 2004, J. Atmos. Sol. Terr. Phys., 66, 167.

Tsurutani, B.T., Gonzales, W.D., Gonzales, A.L.C., Guarnieri, F., Gopalswamy, N., Grande, M., Kamide, Y., Kasahara, Y., Lu, G., Mann, I., McPherron, M., Soraas, F., Vasiliunas, V.: 2006, J. Geophys. Res., 111, A07S01, doi:10.1029/2005JA011273.

Turner, N.E., Cramer, W.D., Earles, S.K., Emery, B.A.: 2009, J. Atmos. Sol. Terr. Phys., 71, 1023.

Wang, H., Lühr, H., Ma, S.Y., Ritter, P.: 2005, Ann. Geophys., 23, 2069-2079. doi:10.5194/angeo-23-2069-2005.

Weatherwax, A.T., Rosenberg, T.J., Maclennan, C.G., Doolittle, J.H.: 1997, Geophys. Res. Lett. 24, 579-582.

Yahnin, A.G., Despirak, I.V., Lubchich, A.A., Kozelov, B.V.: 2004, In: Ganushkina N. and Pulkkinen T. (eds) Proceedings of the 7th International Conference on Substorms, Conference on Substorms, Levi, Finland, 21-27 March 2004 (Reports No. 2004:5, Finnish Meteorological Institute, Helsinki 2004), 3133.

Yermolaev, Y.I., Lodkina, I.G., Nikolaeva, N.S., Yermolaev, M.Y.: 2014, J. Geophys. Res., 119(10), 8126-8136. doi:10.1002/2014JA019826.

Yermolaev, Yu.I., Nikolaeva, N.S., Lodkina, I.G., Yermolaev, M.Yu.: 2009, Cosmic Research (Engl. Transl.), 47, 81-94.

Yermolaev, Y.I., Nikolaeva, N.S., Lodkina, I.G., Yermolaev, M.Y.: 2010, Ann. Geophys., 28., 2177. 